\title{
Pediatric cancer survivorship: is our work nearly done or just beginning?
}

\begin{abstract}
"...the success of curative therapy may be bittersweet for many who struggle with late-occurring chronic health issues resulting from the cancer or its therapy ... approximately $75 \%$ of adults surviving pediatric cancer will develop at least one chronic health problem by 40 years of age."
\end{abstract}

Owing to the significant advances achieved in pediatric cancer therapy over the last 50 years, long-term survival is a reality for $80 \%$ of children and adolescents diagnosed with cancer [1]. However, the success of curative therapy may be bittersweet for many who struggle with lateoccurring chronic health issues resulting from the cancer or its therapy. Recent research indicates that approximately $75 \%$ of adults surviving pediatric cancer will develop at least one chronic health problem by 40 years of age, and $40 \%$ will experience a chronic condition that is severe, life-threatening or fatal $[2,3]$. Cancer-related late effects manifest as a variety of chronic medical and psychosocial disorders that can adversely impact overall health status of the long-term survivor [4-7]. Remarkably, for most outcomes studied, survivors exhibit impressive resilience, and serious life-threatening morbidity is limited to a minority of survivors. However, without intervention, chronic or subclinical changes that persist after cancer treatment can diminish survivors' quality of life and predispose them to early mortality $[6,8]$.

Over the years, recognition of late effects has prompted many therapeutic modifications of pediatric cancer care that have significantly reduced the incidence of acute life-threatening events. In most cases, late treatment sequelae can be anticipated based on therapeutic exposures, but their risk and manifestation in an individual patient is influenced by a myriad of cancer- and host-related factors [9]. Elucidation of factors influencing risk for specific outcomes related to the individual survivor (sex, race/ethnicity, age at diagnosis and attained age), sociodemographic status (education, household income and health insurance) and cancer history (diagnosis, treatment and time from diagnosis) has facilitated identification of groups at highest risk of morbidity [10]. Cancer patients may present with premorbid health conditions that affect their tolerance to therapy and enhance the risk of treatment toxicity [11-13]. Genetic or familial characteristics may confer additional risks for adverse outcomes [14-18]. Treatment modalities and intensity are determined by cancer-related factors including tumor location, histology and biology. Complications experienced during and after cancer therapy may add further morbidity. Cancer-related effects on psychosocial outcomes, including educational achievement, employment status and household income, influence a survivor's access to health insurance, healthcare and remedial services [5,19-21]. Survivors with cancerrelated subclinical organ dysfunction may experience an earlier onset or accelerated progression of health conditions commonly associated with aging. Finally, lifestyle issues such as tobacco and alcohol use, sun exposure, dietary practices, and physical activity may augment the risk of specific health problems predisposed by cancer treatment. Appreciation of the multifactorial nature of cancer-related morbidity is important in devising the optimal plan for health screening and risk-reduction interventions for individual childhood cancer survivors [22].

"Identification of survivors
at risk for adverse health
outcomes permits interventions
to detect, rehabilitate or
prevent morbidity."

Despite the substantial research published concerning childhood cancer outcomes, significant knowledge deficits exist regarding important areas of long-term survivor health. In particular, we are limited in our understanding about the following issues of importance to adult survivors of pediatric malignancies:

- How does cancer-related morbidity affect the natural course of organ senescence during adulthood?
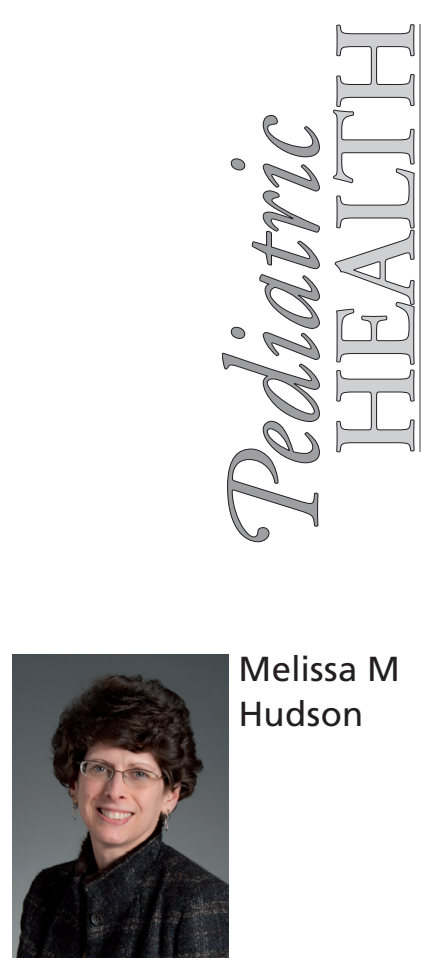

St Jude Children's Research Hospital, Department of Oncology, Cancer Survivorship Division, 262 Danny Thomas Place, Mailstop 735, Memphis,

TN 38105, USA

and

University of Tennessee College

of Medicine, Memphis, TN 38163, USA

Tel.: +19015953300

Fax: +1901595 5845

melissa.hudson@stjude.org

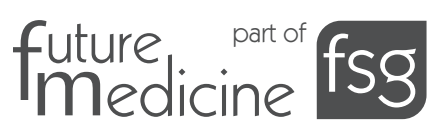


- What are the long-term implications of subclinical effects on cardiovascular, pulmonary, hepatic, musculoskeletal, dental and urinary tract function after contemporary risk-adapted treatment?

- What are the late health outcomes impacting organ function, including neurocognitive, neurosensory, gonadal and sexual function?

- How effective is contemporary radiation technology in reducing radiation-associated toxicity?

- Does combined modality therapy enhance the risk of organ toxicity?

- What are the long-term complications associated with surgical modalities such as amputation, limb-sparing or organ-preserving procedures and their impact on functional status and quality of life?

- What are the late health outcomes resulting from more intensive interventions such as hematopoietic cell transplantation undertaken for children with relapsed and high-risk malignancies?

- What lifestyle factors, such as smoking, obesity, and excessive alcohol intake, exacerbate the conditions mentioned above?

- How do genetic factors influence the risk and manifestation of cancer-related toxicity?

Identification of survivors at risk for adverse health outcomes permits interventions to detect, rehabilitate or prevent morbidity. Continued research is needed to facilitate timely identification of at-risk survivors and evaluate the multifactorial contribution of host, cancer, genetic, health behaviors and aging to the risk of adverse cancer-related health outcomes. This is a challenging endeavor because risk factors are constantly evolving with the introduction of new agents, modification of treatment approaches, and the aging of survivors. Therefore, health outcomes research objectives must adapt as cancer therapies evolve and new risk profiles for cancer-related morbidity are identified. To accomplish these objectives, research targeting well characterized clinical cohorts of adults surviving childhood cancer must be prioritized to improve understanding regarding host- and cancer-related risk factors predisposing to longterm cancer-related morbidity. Knowledge gained from these investigations offers the potential to improve quality of life for cancer survivors and their families, guide healthcare providers developing new treatment approaches and monitor long-term survivors, and facilitate approval of screening and remedial services by insurance companies and legislators.

Without a doubt, our work in pediatric cancer survivorship is just beginning.

\section{Financial \& competing interests disclosure}

This work was supported in part by the Cancer Center Support (CORE) grants CA 21765 and CA 55727 from the National Cancer Institute and by the American Lebanese Syrian Associated Charities (ALSAC). The author has no other relevant affiliations or financial involvement with any organization or entity with a financial interest in or financial conflict with the subject matter or materials discussed in the manuscript apart from those disclosed.

No writing assistance was utilized in the production of this manuscript.

\section{Bibliography}

1. Jemal A, Siegel R, Ward E et al:: Cancer statistics, 2009. CA Cancer J. Clin. 59(4), 225-249 (2009).

2. Oeffinger KC, Mertens AC, Sklar CA et al.: Chronic health conditions in adult survivors of childhood cancer. N. Engl. J. Med. 355(15), 1572-1582 (2006)

3. Geenen MM, Cardous-Ubbink MC, Kremer LC et al:: Medical assessment of adverse health outcomes in long-term survivors of childhood cancer. JAMA 297(24), 2705-2715 (2007).

4. Diller L, Chow EJ, Gurney JG et al.: Chronic disease in the Childhood Cancer Survivor Study cohort: a review of published findings. J. Clin. Oncol. 27(14), 2339-2355 (2009).
5. Hudson MM, Mertens AC, Yasui Y et al.: Health status of adult long-term survivors of childhood cancer: a report from the Childhood Cancer Survivor Study. JAMA 290(12), 1583-1592 (2003).

6. Ness KK, Hudson MM, Ginsberg JP et al:: Physical performance limitations in the Childhood Cancer Survivor Study cohort. J. Clin. Oncol. 27(14), 2382-2389 (2009).

7. Zeltzer LK, Recklitis C, Buchbinder D et al.: Psychological status in childhood cancer survivors: a report from the Childhood Cancer Survivor Study. J. Clin. Oncol. 27(14), 2396-2404 (2009).

8. Armstrong GT, Liu Q, Yasui Y et al.: Late mortality among 5-year survivors of childhood cancer: a summary from the
Childhood Cancer Survivor Study. J. Clin. Oncol. 27(14), 2328-2338 (2009).

9. Oeffinger KC, Hudson MM: Long-term complications following childhood and adolescent cancer: foundations for providing risk-based health care for survivors. CA Cancer J. Clin. 54(4), 208-236 (2004).

10. Hudson MM, Mulrooney DA, Bowers DC et al:: High-risk populations identified in Childhood Cancer Survivor Study investigations: implications for risk-based surveillance. J. Clin. Oncol. 27(14), 2405-2414 (2009).

11. Hyman SL, Gill DS, Shores EA et al:: Natural history of cognitive deficits and their relationship to MRI T2-hyperintensities in NF1. Neurology 60 (7), 1139-1145 (2003). 
12. Ross JA, Spector LG, Robison LL et al:: Epidemiology of leukemia in children with Down syndrome. Pediatr. Blood Cancer 44(1), 8-12 (2005).

13. Trobaugh-Lotrario AD, Smith AA, Odom LF: Vincristine neurotoxicity in the presence of hereditary neuropathy. Med. Pediatr. Oncol. 40 (1), 39-43 (2003).

14. Malkin D, Friend SH, Li FP et al.: Germ-line mutations of the $\mathrm{p} 53$ tumor-suppressor gene in children and young adults with second malignant neoplasms. N. Engl. J. Med. 336(10), 734 (1997).

15. Ross JA, Oeffinger KC, Davies SM et al:: Genetic variation in the leptin receptor gene and obesity in survivors of childhood acute lymphoblastic leukemia: a report from the Childhood Cancer Survivor Study. J. Clin. Oncol. 22(17), 3558-3562 (2004).
16. Relling MV, Rubnitz JE, Rivera GK et al.: High incidence of secondary brain tumours after radiotherapy and antimetabolites. Lancet 354(9172), 34-39 (1999).

17. Relling MV, Yang W, Das S et al.: Pharmacogenetic risk factors for osteonecrosis of the hip among children with leukemia. J. Clin. Oncol. 22(19), 3930-3936 (2004).

18. Wong FL, Boice JD, Abramson DH et al.: Cancer incidence after retinoblastoma. Radiation dose and sarcoma risk. JAMA 278(15), 1262-1267 (1997).

19. Pui $\mathrm{CH}$, Cheng $\mathrm{C}$, Leung W et al.: Extended follow-up of long-term survivors of childhood acute lymphoblastic leukemia. $N$. Engl. J. Med. 349(7), 640-649 (2003).

20. Nathan PC, Greenberg ML, Ness KK et al.: Medical care in long-term survivors of childhood cancer: a report from the childhood cancer survivor study. J. Clin. Oncol. 26(27), 4401-4409 (2008).
21. Oeffinger KC, Mertens AC, Hudson MM et al:: Health care of young adult survivors of childhood cancer: a report from the Childhood Cancer Survivor Study. Ann. Fam. Med. 2(1), 61-70 (2004).

22. Hudson MM: Survivors of childhood cancer: coming of age. Hematol. Oncol. Clin. North Am. 22(2), 211-231, v-vi (2008).

\section{Affiliation}

- Melissa M Hudson St Jude Children's Research Hospital, Department of Oncology, Cancer Survivorship Division, 62 Danny Thomas Place, Mailstop 735, Memphis, TN 38105, USA and University of Tennessee College of Medicine, Memphis, TN 38163, USA

Tel.: +19015953300

Fax: +1 9015955845

melissa.hudson@stjude.org 